\title{
Immunization During the COVID-19 Pandemic: Recommendations From Indian Academy of Pediatrics Advisory Committee on Vaccines and Immunization Practices
}

\author{
Srinivas G Kasi, ${ }^{1}$ Shashi Kant Dhir, ${ }^{2}$ Sanjay Verma, ${ }^{3}$ Harish Kumar Pemde, ${ }^{4}$ S Balasubramanian, 5 \\ Sunil Agarwalla, ${ }^{6}$ AbHay K Shah, ${ }^{7}$ S Shivananda, ${ }^{8}$ Sanjay Marathe, ${ }^{9}$ Kripasindhu Chatarjee, ${ }^{10}$ \\ Sanjay Srirampur, ${ }^{11}$ Srinivas Kalyani, ${ }^{12}$ BakUl J Parekh, ${ }^{13}$ GV Basavaraja ${ }^{14}$ And Piyush Gupta ${ }^{15}$ \\ From ${ }^{1}$ Kasi Clinic, Bengaluru, Karnataka; ${ }^{2}$ Departments of Pediatrics, Guru Gobind Singh Medical College, Faridkot, Punjab; \\ ${ }^{3}$ Department of Pediatrics, Postgraduate Institute of Medical Education and Research, Chandigarh; ${ }^{4}$ Lady Hardinge Medical \\ College, New Delhi, ${ }^{5}$ Department of Pediatrics, Kanchi Kamakoti Childs Trust Hospital, Chennai, Tamil Nadu; ${ }^{6}$ MKCG MCH, \\ Berhampur, Odisha; ${ }^{7}$ Dr Abhay K Shah Children Hospital, Ahmedabad, Gujarat; ${ }^{8}$ Fortis Hospital, Bengaluru, Karnataka; \\ ${ }^{9}$ Marathe Child Care Hospital, Nagpur, Maharashtra; ${ }^{10}$ Gouri Devi Institute of Medical Science and Hospital, Durgapur, West \\ Bengal; ${ }^{11}$ Aditya Super speciality Hospital, Hyderabad, Telangana, ${ }^{12}$ Niloufer Hospital, Osmania Medical College, Hyderabad; \\ ${ }^{13}$ Bakul Parekh Hospital for Children, Mumbai; ${ }^{14}$ IGICH, Bengaluru, Karnataka; and ${ }^{15}$ University College of Medical Sciences, \\ New Delhi; India, for the Advisory Committee on Vaccines and Immunization Practices (ACVIP), 2020-21, Indian Academy of \\ Pediatrics, India. \\ Correspondence to: Dr Srinivas G Kasi, Convener, ACVIP of IAP, Kasi Clinic, 2nd Cross, 3rd Block, Jayanagar, Bengaluru, \\ Karnataka,India.sgkasi@gmail.com
}

During the severe acute respiratory syndrome coronavirus 2 (SARS-CoV-2) pandemic, immunization practices of all age groups, especially routine childhood vaccines, have been interrupted. Immunization is considered an essential health activity, which needs to be resumed as early as possible. This pandemic has created several unique issues related to routine immunization of individual children at clinics, which needs to be addressed. In this communication, the Advisory Committee on Vaccines and Immunization Practices (ACVIP) of Indian Academy of Pediatrics addresses the common questions and issues related to SARS-CoV-2 and routine immunization services. This also includes the recommendations for routine immunization of SARS-CoV-2 suspect and positive children, and for the logistics to be followed for immunization services.

Keywords: COVID-19, Guidelines, Missed Vaccination, Routine immunization.

$\mathrm{T}$ he coronavirus disease (COVID-19) pandemic has negatively impacted every aspect of life. The economic sector has been the most affected and preventive health services have been almost kept on hold. Primary health care services, including immunization, have been disrupted, putting women and children at risk for vaccine-preventable diseases (VPDs), such as measles, rotavirus, and tetanus. Several districts and blocks in India have had partial or complete lockdown on-and-off, affecting movement of people. Fear of getting the infection, social distancing norms and other infection prevention control practices have adversely affected health seeking behavior and routine visits to health care facilities. The primary focus of public health has been diverted towards preparedness and containment of COVID-19 pandemic in the country, and all other preventive health activities have been relegated to the background.

The immunization services had to bear the brunt of these unprecedented circumstances and various on-site and community immunization services were severely affected. In April 2020, the health management and information system data reported a decrease in the number of routine immunization sessions relative to the previous year. The number of fully immunized children also decreased over the same time period. It is to be emphasized that any flare of VPDs will additionally burden already stressed health care systems.

The severe acute respiratory infection 2 (SARS-CoV2) pandemic has created several unique issues related to vaccines and immunization services. ACVIP has taken on itself to address the common questions and issues related to COVID-19 and routine immunization services in a question-answer format, with an objective to guide pediatricians on these important issues.

\section{Should children be vaccinated during the pandemic?}

ACVIP recommends that all routine vaccinations be administered as scheduled, even during the COVID-19 
pandemic as it is an essential health activity [1]. The benefits of immunizations far outweigh the associated risks. The importance of continued immunization activities is further emphasized by the observation that during the 2014-2015 Ebola outbreak, the increased number of deaths caused by measles, malaria, HIV/AIDS and tuberculosis attributable to health system failures, exceeded deaths from Ebola itself. In a benefit-risk analysis of health benefits versus excess risk of SARSCoV-2 infection, in Africa, it was estimated that in a highmortality scenario, for every one excess COVID-19 death attributable to SARS-CoV-2 infections acquired during routine vaccination clinic visits, 84 (95\% CI 14-267) deaths in children could be prevented by sustaining routine childhood immunization. The advantages of sustained immunizations extended to their siblings $(<20$ years) and to other family members also [2]. In the private practice settings, the existing ACVIP Guidelines are to be followed for routine immunization.

Is my child at an increased risk for getting infected with SARS-CoV-2 by the hospital/clinic visit for immunization?

If SMS (social distancing, mask, sanitization) is strictly followed, and the recommended COVID-19 related norms are observed in the immunization session, the risk is minimal.

\section{Is there any risk from immunizing a child during the pandemic?}

There is no documented risk of immunizing a well child during the COVID-19 pandemic. COVID-19 is still an evolving disease and hence we need to monitor strictly for any increased adverse events following immunization (AEFI).

\section{Is there a risk if we vaccinate a child during the incubation period of COVID-19?}

Currently there is no evidence that there is any risk to the vaccinee if vaccination is done during the incubation period of COVID-19. The efficacy and safety of the administered vaccines would be the same.

\section{Does vaccination increase a child's risk of becoming infected with SARS-CoV-2 or of developing COVID-19?}

In general, vaccination against one disease does not weaken the immune response to another disease. As of now, there is no evidence that vaccination would increase the risk of a child becoming infected with COVID-19 or affect the course of the disease in a child who has been inadvertently vaccinated during the asymptomatic phase or incubation period [3].
Is there a change in the existing immunization schedule during the pandemic?

There is no information about the effect of COVID-19 on responses following immunization. ACVIP recommends that in private practice settings, the existing ACVIP Guidelines are to be followed for routine immunization [4]. There is currently no need for a change in the immunization schedule.

How is area categorization being done by the Government during the COVID-19 pandemic for providing immunization services?

Based on World Health Organization (WHO) guidelines, the Government of India (GOI) has categorized areas (district/sub-division/municipal corporation/ward/any other appropriate administrative unit) into Red and Orange zones with active COVID-19 cases, and Green zones with no active COVID-19 cases [5]. Areas where COVID-19 cases are reported and surrounding areas with risk of COVID-19 spread are classified as Containment zone and Buffer zone respectively; whereas areas outside the buffer zone are identified as Area beyond buffer zone. The categorization of containment and buffer zones is a dynamic process updated on a weekly basis or earlier. In alignment with the area categorization, immunization services are classified into two heads: Immunization in containment and buffer zones, and immunization in areas beyond buffer zones and green zones.

\section{How are immunization services to be conducted in containment zones and buffer zones?}

Traditionally, immunization services in India get delivered through the following modes: Birth dose vaccination (at delivery points), Health facility-based sessions (at fixed health facilities), and Outreach sessions (as part of Urban/village health sanitation and nutrition day services). The birth dose vaccination at all health facilities should be provided to all the eligible babies. A child reporting to the health care facility due to any reason should not be denied immunization and every opportunity must be utilized for vaccinating the beneficiaries. The health facility-based immunization services should be provided only on demand to walk-in beneficiaries. The outreach immunization sessions should not be undertaken in these areas. However, the facility-based as well as outreach immunization activities can be started after two weeks of delisting of the area as containment or buffer zone, after being assessed for the COVID-19 risk by the district authorities [6].

What are current recommendations for immunization services in the area beyond buffer zone and green zone? 
All areas beyond the buffer zone and in the green zone need to follow similar guidelines. In these areas, the health facility-based immunization services should be provided to all the beneficiaries. The outreach (modified) immunization sessions can also be undertaken at a predetermined site having adequate space and in a modified way. Such a modified outreach session is to be planned for less than 500 people, with a number of beneficiaries not more than 10-15 per session. Less than five persons should be present at the session site while maintaining a distance of 1 meter from each other. Organization of such sessions will be at the discretion of the district administration, with clear planning for social distancing and hand washing at session site [6].

\section{Should children from containment or buffer zones be vaccinated if they report for vaccination in a facility outside those areas?}

Since the residents of the containment or buffer zones are considered as suspects, they should not be encouraged to go out of containment zones, as per government instructions. Active immunization activities are not allowed in such areas. However, when any child from such areas reaches the healthcare facilities, he/she should be offered immunization as per the requirement of the child.

What logistics are required while setting up immunization practices during COVID-19 times?

The logistics may be divided into preparation of the space, personnel, vaccinee, maintaining the waiting area and post vaccination care (Box I).

\section{What should be done if certain due vaccines are missed?}

This is a common situation in the COVID-19 pandemic. The parents have to be reassured that the vaccination schedule can be resumed without any need to restart the series. Multiple vaccines in one sitting and using the minimum permitted interval between two doses of the same inactivated vaccine can be practiced to complete the schedule in the shortest possible time.

The vaccination services should be restarted as early as possible. Missed vaccines have put the society at an increased risk of VPDs. Multiple epidemics of measles occurred in 2015 in Guinea because of the interrupted immunization during the Ebola outbreak [7]. The vaccine provider should track the cohort of children who have missed the vaccine and immunize them, as soon as the vaccination becomes feasible. Public awareness should also be done to sensitize them about the catch-up vaccination. The parents should be reassured; that there is a window period in which the vaccines could be given; and once given, it would have similar efficacy in future. Following principles need to be followed:

- The birth dose of hepatitis B vaccine should be administered within 24 hours of birth and OPV, and BCG vaccines should be given as early as possible after birth. If for any reason this is not done, these vaccines should be administered at the first contact with the healthcare facility.

- The primary vaccination series and the vaccines for outbreak prone diseases should be prioritized for example DPT, hepatitis B, Hib, OPV/IPV, rotavirus, $\mathrm{PCV}$, influenza, varicella and MR/MMR. Postponing these vaccines is to be avoided.

- The pneumococcal and influenza vaccine should also be given to the vulnerable groups.

- The age specific recommendations of giving vaccines e.g. for pneumococcal, meningococcal and rotavirus vaccine should be followed.

- Multiple vaccines can be administered in the same session without fear of any increased adverse effects.

- Typhoid conjugate vaccines may be clubbed with the influenza vaccine at 6 months or MR/MMR at 9 months.

- Inactivated JE vaccines (where applicable) can be administered at 1 year.

- We may use the shortest acceptable interval between two doses of the same vaccine if the prospective vaccinee reports to a health facility; and is unlikely to come for follow-up. For inactivated primary vaccines this is 28 minus 4 days i.e. 24 days.

- The vaccination of healthcare personnel should be up to date in their age appropriate vaccinations.

- When missed (because of the logistic issues of transport etc.), hepatitis A vaccines and HPV vaccines may be administered after the priority vaccines have been given.

- When missed (because of the logistic issues of transport etc.), the booster dose(s) may be given at the next earliest available opportunity.

- If a child is in a healthcare facility for any reason, and eligible for immunization, this opportunity should be utilized for administering eligible vaccines.

What schedule should be followed for vaccination at birth?

The vaccination at birth depends upon the COVID-19 


\section{Box I Logistics for Immunization Preparedness During COVID-19}

\section{Preparation of the vaccination area}

- Exclusive vaccination sessions are recommended in separate vaccination rooms. If the same floor/ building is used for other patients, provision should be made for separate entry and exit paths for the prospective vaccinees to avoid mixing with the general patients.

- Adequate well-ventilated seating space having one meter distancing from another person should be available. The area should have exhaust fans for adequate air circulation, and windows should be kept open.

- Sanitizers, soap and running water should be made available in adequate amounts at the entry point of the vaccination area.

- Vaccination staff, having any Flu like symptoms, should not be allowed to vaccinate.

- It is essential that the doctor and supporting staff utilize adequate PPE. A mask (N95 mask preferably and threelayered surgical mask when N95 is not available), gloves, and face shield along with scrupulous hand hygiene are likely to protect from aerosol generation by a crying child.

- New clean gloves (non-sterile) should be donned before each vaccination.

- Hand hygiene with alcohol-based hand sanitizers containing minimum $60 \%$ alcohol, for a minimum 20 seconds is to be practiced before and after each vaccination. Gloved hand should also be sanitized.

- COVID-19 awareness material should be displayed in the vaccination area.

- Continuous training should be imparted to the health care personnel engaged in vaccination practices.

Vaccinee logistics

- The vaccinee should preferably be called by appointment although no opportunity should be missed for vaccination.

- Overcrowding should be avoided. Physical distancing of 1 meter should be observed.

- Utilize every healthcare visit for immunization, provided there are no precautions/ contraindications and the interval between vaccines are maintained as per published guidelines.

- Minimum number of attendants preferably single (maximum two) should only be allowed in the premises.

- The accompanying individuals should be screened for fever and respiratory symptoms and if symptomatic, they should not be allowed in the vaccination area and should be advised to get examined at the health care services.

- Attendants who are more than 60 years of age or have comorbid conditions should be requested not to accompany the vaccinee.

- All caretakers and children, except infants should wear a triple layer mask and provision should be made for providing these at the entrance of the vaccination area.

- The accompanying persons should be made aware of the social distancing, hand washing/sanitizing and respiratory hygiene during the visit.

Waiting area

- The number of waiting persons both before and after the vaccination should not be more than the capacity of the area while maintaining the distance of $1 \mathrm{~m}$ between two persons.

- Post vaccination, the vaccinee must be observed for 15 minutes for development of any immediate AEFI.

- This waiting period should be used for group counselling. Key preventive messages pertaining to precautions during COVID period, strengthening of hand hygiene, social distancing, breastfeeding, dietary advice, and danger signs could be discussed during this period.

- The informative written material can also be kept in the waiting areas.

Logistics post vaccination

- The furniture used should be thoroughly cleaned with appropriate sanitizers.

- Proper sanitization of the anthropometry equipment should be ensured immediately after each use.

- The biomedical waste generated should be disposed of at source.

- Digital payment is to be encouraged. 
status of mother as well as the neonate, and the clinical condition of the baby [8]. The recommendations are given in Table $\mathbf{I}$.

\section{What is to be done if the vaccinee is suspected or diagnosed to have SARS-CoV-2 infection?}

ACVIP recommends that if the prospective vaccinee has been infected with COVID-19 or is under quarantine, the vaccination is to be done only after the quarantine period is over and the clinical condition of the baby is stable. If the child has any symptoms suggestive of COVID-19 infection, vaccination is to be avoided till the symptoms resolve. It is also emphasized that the presence of fever may interfere in the differentiation between the disease progression and vaccine induced adverse events. Therefore, it is best to wait for the resolution of symptoms before vaccinating in COVID times. Standard guidelines given in IAP guidebook on Immunization 2018-2019 (vaccination in special situations) should be followed if children develop 'Multisystem inflammatory syndrome' during COVID infection and require intravenous immunoglobulin or steroids [9]. It is not mandatory to document a negative COVID test before vaccination.

Do Bacille-Calmette-Guérin (BCG) or MMR vaccines have some role in controlling the ongoing COVID-19 pandemic?

Epidemiological studies have suggested a negative association between national BCG vaccination policy and the prevalence and mortality of COVID-19. Observational studies have also suggested that countries with recent MMR campaigns or established MMR vaccinations in their national programs have generally reported lower mortalities due to COVID 19.

Table I Birth Dose Vaccination Recommendation During COVID-19

\begin{tabular}{|c|c|c|c|c|}
\hline Scenario & Mother & Neonate & Status & Schedule \\
\hline A & $\begin{array}{l}\text { No clinical suspicion of } \\
\text { COVID-19 infection }\end{array}$ & $\begin{array}{l}\text { No clinical suspicion of } \\
\text { COVID-19 infection }\end{array}$ & Normal & $\begin{array}{l}\text { - Hepatitis B: At birth or as early as possible } \\
\text { within } 24 \mathrm{~h} \\
\text { - BCG: At birth or as early as possible } \\
\text { - OPV: At birth or as early as possible } \\
\text { within } 15 \mathrm{~d}\end{array}$ \\
\hline B & $\begin{array}{l}\text { Suspected but COVID } \\
\text { unconfirmed }\end{array}$ & $\begin{array}{l}\text { No clinical suspicion of } \\
\text { COVID-19 infection }\end{array}$ & Normal & Same as above \\
\hline $\mathrm{Ca}$ & COVID positive & $\begin{array}{l}\text { Asymptomatic but not } \\
\text { tested }\end{array}$ & $\begin{array}{l}\text { Contact of } \\
\text { confirmed case }\end{array}$ & Same as above \\
\hline $\mathrm{Cb}$ & COVID positive & $\begin{array}{l}\text { Symptomatic but not } \\
\text { tested }\end{array}$ & $\begin{array}{l}\text { Contact of } \\
\text { confirmed case }\end{array}$ & $\begin{array}{l}\text { - Hepatitis B: At birth or as early as } \\
\text { possible within } 24 \mathrm{~h} \\
\text { - BCG: At time of discharge } \\
\text { - OPV: At time of discharge but less } \\
\text { than } 15 \mathrm{~d}\end{array}$ \\
\hline $\mathrm{Da}$ & COVID positive & $\begin{array}{l}\text { COVID positive but } \\
\text { asymptomatic or mildly } \\
\text { symptomatic }\end{array}$ & $\begin{array}{l}\text { Confirmed case, } \\
\text { capable of trans- } \\
\text { mitting disease }\end{array}$ & $\begin{array}{l}\text { Hepatitis*: At time of discharge but } \\
\text { less than } 15 \mathrm{~d} \\
\text { - BCG: At time of discharge } \\
\text { - OPV: At time of discharge but less than } \\
15 \mathrm{~d}\end{array}$ \\
\hline $\mathrm{Db}$ & COVID positive & $\begin{array}{l}\text { COVID positive but } \\
\text { severely symptomatic }\end{array}$ & $\begin{array}{l}\text { Confirmed case, } \\
\text { capable of trans- } \\
\text { mitting disease }\end{array}$ & $\begin{array}{l}\text { - Hepatitis } \# \text { : At time of discharge but less } \\
\text { than } 15 \mathrm{~d} \\
\text { - BCG: At time of discharge } \\
\text { - OPV: At time of discharge but less than } \\
15 \mathrm{~d}\end{array}$ \\
\hline $\mathrm{E}$ & $\begin{array}{l}\text { No clinical suspicion } \\
\text { of COVID-19 infection }\end{array}$ & $\begin{array}{l}\text { Symptomatic but COVID } \\
\text { status unknown }\end{array}$ & Suspect & $\begin{array}{l}\text { - Hepatitis**: At time of discharge but less } \\
\text { than } 15 \mathrm{~d} \\
\text { - BCG: At time of discharge } \\
\text { - OPV: At time of discharge but less than } \\
15 \mathrm{~d}\end{array}$ \\
\hline
\end{tabular}

*In infants born to HBsAg/HBeAg-positive/Hepatitis B status unknown mother, hepatitis B vaccination to be given within first 24 hours or as soon as

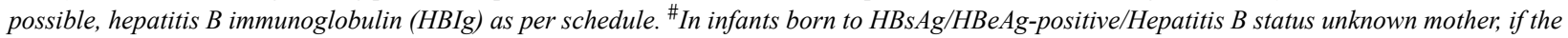
baby is clinically stable, hepatitis $B$ vaccination to be given within first 24 hours or as soon as possible, HBIg to be given as per schedule. Schedule adapted from Ref No 8 . 
As of now, there is no evidence that BCG vaccine has a protective role against occurrence of SARS-CoV-2 infection [10]. Based on the current evidence, ACVIP does not recommend use of BCG or MMR vaccine for the protection of individuals against COVID-19 infection [10-12]. Various attributed potential nonspecific effects of the BCG vaccine in preventing COVID-19 as of now remain a hypothesis, because of multiple confounding factors [13]. Clinical trials are underway and the recommendations would be updated once more evidence is available.

Disclaimer: These guidelines are meant for practicing pediatricians in their office set up or hospital. These guidelines are based on the available knowledge of COVID 19 as on date. Any further guidance based on evolving scenarios will be issued accordingly. Members are informed that these are only recommendations and they should be taken in context with local advisories issued by health authorities in the areas where the member provides medical services.

Contributors: All authors conceived the idea, reviewed the manuscript, analyzed and approved the manuscript. Funding: None; Competing interests: None stated.

\section{REFERENCES}

1. Indian Academy of Pediatrics. Advisory Committee on Vaccines and Immunization Practices (ACVIP). ACVIP Guidelines on Immunization during COVID 19 Pandemic. Accessed September 25, 2020. Available from: https:// iapindia.org/pdf/1455-FINAL-ADVISORY-ACVIPGuidelines-on-Immunisations-during-COVID-19Pandemic-skd.pdf

2. Abbas K, Procter SR, van Zandvoort K, et al. Routine childhood immunisation during the COVID-19 pandemic in Africa: A benefit-risk analysis of health benefits versus excess risk of SARS-CoV-2 infection. Lancet Glob Health. 2020;8:e1264-e72.

3. World Health Organization. Q\&A on vaccination during the COVID-19 pandemic. Accessed October 2, 2020. Available from: https://www.euro.who.int/en/healthtopics/disease-prevention/vaccines-and-immunization/qand-a-on-vaccination-during-the-COVID-19-pandemic.
4. Balasubramanian S, Shah A, Pemde HK, et al. Indian Academy of Pediatrics (IAP) Advisory Committee on Vaccines and Immunization Practices (ACVIP) Recommended Immunization Schedule (2018-19) and Update on Immunization for Children Aged 0 Through 18 Years. Indian Pediatr. 2018;55:1066-74.

5. World Health Organization. Guiding principles for immunization activities during the COVID-19 pandamic. Accessed September 25, 2020. Available from: https:// apps.who.int/iris/handle/10665/331590

6. Government of India. Ministry of Health and Family Welfare. Immunization Services During and Post Covid-19 Outbreak. Accessed September 25, 2020. Available from: https://www.mohfw.gov.in/pdf/3 Immunization Servicesduring COVIDOutbreak Summary150520202. pdf

7. Suk JE, Jimenez AP, Kourouma M, Derrough T, Baldé M, Honomou P. Post-Ebola measles outbreak in Lola, Guinea, January-June 2015. Emerg Infect Dis. 2016;22:1106-8.

8. Vaccination of Newborns in the Context of the COVID-19 Pandemic, 19 May 2020 - PAHO/WHO. Pan American Health Organization. Accessed October 2, 2020. Available from: http://www.paho.org/en/documents/vaccinationnewborns-context-COVID-19-pandemic-19-may-2020/

9. World Health Organization. Bacille Calmette-Guérin (BCG) vaccination and COVID-19. Scientific Brief, 12 April 2020. Accessed on October 9, 2020. Available from: https://www.who.int/news-room/commentaries/detail/ bacille-calmette-gu\%C3\%A9rin-(bcg)-vaccination-andCOVID-19

10. Indian Academy of Pediatrics (IAP). IAP Guidebook on Immunization. Accessed October 9, 2020. Available from: https://iapindia.org/iap-guidebook-on-immunization/

11. Vashishtha VM. Are BCG-induced non-specific effects adequate to provide protection against COVID-19? [published online ahead of print, 2020 Aug 07]. Hum Vaccin Immunother. 2020.

12. Deshpande S, Balaji S. MMR vaccine and COVID-19: A myth or a low risk-high reward preventive measure? Indian Pediatr. 2020;57:773.

13. Dinleyici EC, Borrow R, Safadi MAP, van Damme P, Munoz FM. Vaccines and routine immunization strategies during the COVID-19 pandemic [published online ahead of print, 2020 Aug 26]. Hum Vaccin Immunother. 2020;1-8. 